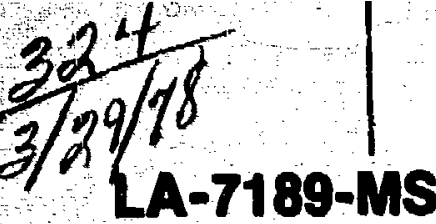

Informal Report
UC.32 and UC.34

Issued: March 1978

\title{
Cost of Splitting in Monte Carlo Transport
}

\author{
C. J. Everett \\ E. D. Cashwell
}




\title{
COST OF SPLITTING IN
}

MONTE CARLO TRANSPORT

by

C. J. Everett and E. D. Cashwell

\begin{abstract}
In a simple transport problem designed to estimate transmission through a plane slati of $X$ free paths by Monte Carlo methods, it is shown that $m-s$ litting $(m \geq 2)$ does not pay unless $\mathrm{e}^{X}>\mathrm{m}(\mathrm{m}+3) /(\mathrm{m}-1)$. In such a case, the minimum total cost in terms of machine time is obtained as a function of $m$, and the optimal value of $m$ is determined.
\end{abstract}

I. INTRODUCTION

If an elementary experiment can result in $m+1$ different ways: $i=0, \ldots, m$, the $i$ 'th way having probability $p(i)$ and an assigned "weight" $x(i)$, then

$$
\bar{x}=\sum_{i=0}^{m} x(i) p(i)
$$

is called the "expected value" of $x$, and

$$
v=\overline{x^{2}}-\bar{x}^{2}
$$

This report was prepared as in eccoutl: of wosk spensored by the United States Govemment. Ne:'ther the United Stales nor the United Sutes Department of Energy, nor any of thetr employets, nor any or their contractori, subconisactors, or their employees, makes any warranty, express or implied, or assumes any legal liability of reaponsiblity for the sccuracy. cutspletenexs or usefulnexs of any information, apparatus, product or p:ocest disclosed, or mpresents that its un would not

its "variance", where $\overline{x^{2}}=\sum_{i=0}^{m} x^{2}(i) p(i)$.

In sucti a case, the central limit theorem asserts that, in a large number $N$ of such experiments, 


$$
P\left|\frac{\left(\sum_{\tau_{i=1}}^{N} \times\left(i_{\tau}\right) / N\right)-\bar{x}}{\bar{x}}\right|<z \cdot \frac{\sqrt{V / N}}{\bar{x}} \mid \simeq \frac{2}{\sqrt{2 \pi}} \int_{0}^{z} e^{-t^{2} / 2 d t} .
$$

The probability $P$ here means $\sum p\left(i_{1}\right) \ldots p\left(i_{N}\right)$ summed over all those $N$-tuples $\left(i_{1}, \ldots, i_{N}\right)$ for which the sum $x\left(i_{1}\right)+\ldots+x\left(i_{N}\right)$ satisfies the stated inequality.

The fraction $E=\frac{\sqrt{V / N}}{\bar{x}}$ is called the "relative error" of the mean $\bar{x}$.

In Monte Carlo transport problems one is concerned with the estimation of such a mean $\bar{x}$ by processing a large number $N$ of source particles. Each of the $N$ "histories" may be regarded as the trial $\tau$ of an elementary expeiriment contributing a certain weight $x\left(i_{\tau}\right)$ to a definite counter with some probability $p\left(i_{\tau}\right)$. Obviously the practical question is the choice of a method of sampling which minimizes the total cost $C$ of processing a fixed number $N$ of source particles, with a stipulated error $E$. To make the ideas and notation clear, we Tist the essential parameters.

$N=$ number of source particles

$\bar{x}=$ expected value of $x$ (method independent, necessarily)

$V=$ variance of $x$ (method dependent)

$E=(V / N)^{1 / 2} / \bar{x}$, stipulated error (method independent)

$c=$ cost per source particle (method dependent)

$C=C N$ total cost of method (method dependent)

We seek a method for which $C$ is minimal. Since $C=c N=c\left(V / E^{2} \bar{x}^{2}\right)$ where $E^{2} \bar{x}^{2}$ is method independent, we need only minimize the "cost function"

$$
f=c V \text {, }
$$

where both factors are method dependent. Note that the number $\mathrm{N}$ of particles is irrelevant.

\section{I1. A SIMPIE TRANSPORT PROBLEM}

We obtain such an optimal method for the following simple problem, the objective being determination of the expected transmission $\bar{x}$. 
a. Source particles enter plane slab normal to surface.

b. Collision results in total absorption.

c. Slab $X$ free paths thick.

d. $c_{0}=$ cost of computing one distance-to-collision (infinite medium) and comparison with distance to boundary.

As options we consider the following methods:

Method I. No splitting.

Method II. Splitting each source particle into $m \geqq 2$ particles, each of weight $1 / m$, at entrance.

Method III. Such splitting at distance $x$ from entrance, $0<x<x$.

III. TOTAL COST OF THE METHODS

In Method I (see Fig. 1), with no splitting, the elementary experiment is a hit $(i=1)$ or miss $(i=0)$ situation, with

$$
\begin{array}{ll}
p(1)=e^{-x} \equiv p & x(1)=1 \\
p(0)=q=1-p & x(0)=0
\end{array}
$$

in the notation of Sec. I. (We use the notation $p=e^{-X}$ throughout.) Hence

$$
\begin{aligned}
& c=c_{0} \\
& \bar{x}=p \\
& \overline{x^{2}}=p \\
& V=p-p^{2}=p q \\
& f_{I}=c V=c_{0} p q
\end{aligned}
$$

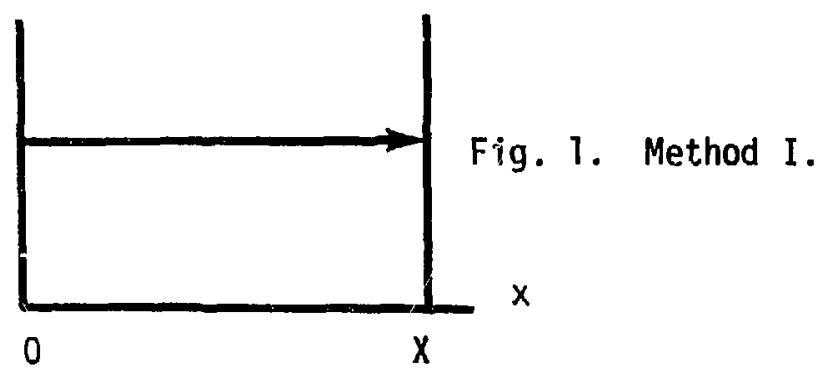

In Method II, with 1-to-m splittiniy at $x=0$, there are $m+1$ possible outcomes to an elementary experiment, namely the arrival at $x=\dot{i}$ of $i$ tracks, $i=0,1, \ldots, m$. Here we have

$$
p(i)=\left(\begin{array}{c}
m \\
i
\end{array}\right) q^{m-i} p i, x(i)=(i / m) i=0, \ldots, m,
$$




$$
\begin{aligned}
c & =m c_{0} \\
\bar{x} & =\sum_{0}^{m}(i / m)\left(\begin{array}{l}
m \\
i
\end{array}\right) q^{m-i} p^{i}=\sum_{1}^{m}\left(\begin{array}{c}
m-1 \\
i-1
\end{array}\right) q^{m-i} p^{i} \\
& =\left(\sum_{0}^{m-1}\left(\begin{array}{c}
m-1 \\
i
\end{array}\right) q^{m-1-i} p^{i}\right) p=(q+p)^{m-1} p=p \\
\overline{x^{2}} & =\sum_{0}^{m}(i / m)^{2}\left(\begin{array}{l}
m \\
j
\end{array}\right) q^{m+i} p^{i}=\sum_{1}^{m}(i / m)\left(\begin{array}{c}
m-1 \\
i-1
\end{array}\right) q^{m-i} p^{i} \\
& =(p / m) \sum_{0}^{m-1}(i+1)\left(_{i}^{m-1}\right) q^{m-1-i} p^{i}=\frac{p}{m}\{(m-1) p+1\} \\
& =p^{2}+\frac{1}{m}\left(p-p^{2}\right)=p^{2}+p q / m \\
V & =p q / m \\
f_{I I} & =\left(m c_{0}\right)(p q / m)=c_{0} p q .
\end{aligned}
$$

Hence, compared with Method I, Method II m-tuples the cost per particle, and cuts the variance by $m$, leaving the function $f$ unchanged.

In Method III with m-splitting at $x, 0<x<x$ in case of arrival at $x$, there are the same $m+1$ possibilities as in Method II, but now

$$
\begin{aligned}
& p(0)=1-e^{-x}+e^{-x} Q^{m}, x(0)=0 . \\
& p(i)=e^{-x}\left(\begin{array}{c}
m \\
i
\end{array}\right) Q^{m-i} p^{i}, x(i)=(i / m), i=1, \ldots, m, \\
& \text { where } P=e^{-(x-x)}=e^{x} p \text { and } Q=1-p .
\end{aligned}
$$

Using the results of Method II in the summations, with $P, Q$ replacing $p, Q$, we obtain for this method

$$
c=c_{0}+e^{-x}\left(m c_{0}\right)=c_{0}\left(1+m e^{-x}\right)
$$

4 


$$
\begin{aligned}
\bar{x} & =\sum_{0}^{m} x(i) p(i)=e^{-x} \sum_{1}^{m}(i / m)\left(\begin{array}{c}
m \\
i
\end{array}\right) q^{m-i} p^{i} \\
& =e^{-x} p=p \\
\overline{x^{2}} & =\sum_{0}^{m} x^{2}(i) p(i)=e^{-x} \sum_{1}^{m}(i / m)^{2}\left(\begin{array}{c}
m \\
i
\end{array}\right) q^{m-i} p^{i} \\
& =e^{-x}\left(p^{2}+p Q / m\right)=p\left(p+\frac{1-p}{m}\right)=\frac{p}{m}\left(1+(m-1) p e^{x}\right) \\
& =p^{2}\left\{\frac{1}{m p}+\frac{m-1}{m} e^{x}\right\} \\
V & =p^{2}\left\{\left(\frac{1}{m p}-1\right)+\frac{m-1}{m} e^{x}\right\} \\
& =c_{0} p^{2}\left(1+m e^{-x}\right)\left\{\left(\frac{1}{m p}-1\right)+\frac{m-1}{m} e^{x}\right\} \\
& =c_{0} p^{2}\left\{\left(\frac{1}{m p}+(m-2)\right)+\frac{m-1}{m} e^{x}+\left(\frac{1}{p}-m\right) e^{-x}\right\}, 0<x<x .
\end{aligned}
$$

This indicates the total cost of splitting at an interral surface $x$ free paths from entry, as compared with $f_{I}=c_{0} p q$ of Method $I$.

IV. CASE OF INCREASING $f(x)$ IN METHOD III

From (1) we see that the derivative

$$
\begin{aligned}
f^{-\prime}(x) & =c_{0} p^{2}\left\{\frac{m-1}{m} e^{x}-\left(\frac{1}{p}-m\right) e^{-x}\right\} \\
& =c_{0} p^{2} e^{-x}\left\{\frac{m-1}{m} e^{2 x}-\frac{1}{p}+m\right\} .
\end{aligned}
$$

Hence, if $e^{X}=\frac{1}{p} \leqslant m+\frac{m-1}{m}$, then

$$
f^{\prime}(x)>c_{0} p^{2} e^{-x}\left\{\frac{m-1}{m}-\frac{1}{p}+m\right\} \geqslant 0,0<x<x,
$$


$f(x)$ is strictly increasing, and from (1)

$\lim _{x \rightarrow 0^{+}} f(x)=c_{0} p^{2}\left\{\frac{1}{m p}+(m-2)+\frac{m-1}{m}+\frac{1}{p}-m\right\}$

$=c_{0} p^{2}\left\{\frac{m+1}{m} \frac{1}{p}-\frac{m+1}{m}\right\}=\frac{m+1}{m} c_{0} p q=\frac{m+1}{m} f_{I}>f_{I}$.

Hence, if $e^{X} \leq m+\frac{m-1}{m}$, splitting never pays.

v. CASE OF $f(x)$ CONCAVE-UP IN METHOD II:

We now assume $e^{X}=\frac{1}{p}>m+\frac{m-1}{m}$. In such a case, we see from (2) that

$f^{-}(0)=c_{0} p^{2}\left\{\frac{m-1}{m}-\frac{1}{p}+m\right\}<0$

$f^{\prime}(x)=c_{0} p^{2} e^{-x}\left\{\frac{m-1}{m} e^{2 x}-e^{x}+m\right\}>0$

(since the quadratic has discriminant $b^{2}-4 a c=1-4(m-1)=5-4 m \leqslant 0$ for $m \geqslant 2$ ). Moreover

$f^{\prime \prime}(x)=c_{0} p^{2}\left\{\frac{m-1}{m} e^{x}+\left(\frac{1}{p}-m\right) e^{-x}\right\}>0$, since $\frac{1}{p}>m+\frac{m-1}{m}>m$.

Hence $f(x)$ has a minimum at a unique $x_{0}$ where from $(2), e^{2 x_{0}}=\frac{m}{m-1}\left(\frac{1}{p}-m\right)$;

$0<x_{0}<x$.

Thus there is a possibility that $f\left(x_{0}\right)$ might be less than $f_{I}$ in this case.

We next compute from (1), the minimal value

$$
\begin{aligned}
f\left(x_{0}\right) & =c_{0} p^{2} e^{x_{0}}\left\{\left(\frac{1}{m p}+(m-2)\right) e^{-x_{0}}+\frac{m-1}{m}+\left(\frac{1}{p}-m\right) e^{-2 x_{0}}\right\} \\
& =c_{0} p^{2} e^{x_{0}}\left\{\left(\frac{1}{m p}+(m-2)\right) e^{-x_{0}}+\frac{m-1}{m}+\frac{m-1}{m}\right\} \\
& =c_{0} p^{2}\left\{\left(\frac{1}{m p}+(m-2)\right)+2 \frac{m-1}{m} e^{x_{0}}\right\}
\end{aligned}
$$




$$
=c_{0} p\left\{\frac{1}{m}+(m-2) p+2 p\left(\frac{m-1}{m}\left(\frac{1}{p}-m\right)\right)^{1 / 2}\right\}
$$

This is the minimal cost of m-splitting in the case $e^{x}=\frac{1}{p}>m+\frac{m-1}{m}$.

To compare this with $f_{I}$, we consider the ratio

$\rho=f\left(x_{0}\right) / c_{0} p q=\frac{\frac{1}{m}+(m-2) p+2 p\left(\frac{m-1}{m}\left(\frac{1}{p}-m\right)\right)^{1 / 2}}{1-p}$.

It is easy to rerify the equivalence of the following relations:

$p<1$

$\frac{1}{m}+(m-2) p+2 p\left(\frac{m-1}{m}\left(\frac{1}{p}-m\right)\right)^{1 / 2}<1-p$

$2\left(\frac{m-1}{m}\left(\frac{1}{p}-m\right)\right)^{1 / 2}: \frac{m-1}{m}\left(\frac{1}{p}-m\right)$

$\left.0<\left(\frac{m-1}{m}\right)^{2}\left(\frac{1}{p}-m\right): \frac{1}{p}-\frac{m(m+3)}{m-1}\right)$

Hence, for $e^{X} \equiv \frac{1}{p}>i n+\frac{m-1}{m}$, m-splitting at the optimal place $x_{0}$ costs less than Method I iff

$$
e^{x} \equiv \frac{1}{p}>\frac{m(m+3)}{m=1}
$$

(Note that $\left.m(m+3) /(m-1)>m+\frac{m-1}{m}\right)$

For fixed $m$, and $X \rightarrow \infty, p=e^{-X} \rightarrow 0$, one sees from (5) that $\rho \rightarrow 1 / m$.

VI. THE OPTIMAL VALUE OF $\mathrm{m}$

So far, we have held $m \geqslant 2$ fixed, and shown that m-splitting at the optimal position $x_{0}$ pays iff the number $X$ of slab free paths is sufficiently large, namely 


$$
e^{X} \equiv 1 / p>m(m+3) /(m-1) \equiv R(m)
$$

The function $R(m)$ has the values

\begin{tabular}{l|l}
$\mathrm{m}$ & $\mathrm{R}(\mathrm{m})$ \\
\hline 2 & 10 \\
3 & 9 \\
4 & $91 / 3$ \\
5 & 10 \\
6 & $104 / 5$
\end{tabular}

with minimum 9 at $m=3$. Hence, given $X$ we have the cases; for $\frac{1}{p} \equiv e^{X}$,

1. $1 / p \leqslant 9$, splitting never pays.

2. $9<1 / p \leqslant 91 / 3$, only $m=3$ pays.

3. $91 / 3<1 / p \leqslant 10$, only $m=3$ and $m=4$ pay.

4. $10<1 / p, m=2,3,4,5$ pay, as well as any other $m$ for which $m(m+3) /(m-1)$ $<1 / p$.

Hence for any given $x$, the set of integers $m \geqslant 2$ for which $m-s p l i t$ ting pays is well defined, and the remaining problem is to determine which $m$ of this set yields a least total cost, namely

$$
f\left(x_{0}\right) \equiv g(m)=c_{0} p^{2}\left\{m-2+\frac{1}{m p}+2\left(\frac{m-1}{m} \cdot \frac{1}{p}-(m-1)\right)^{1 / 2}\right\}
$$

as given by (4).

For case 3 above the decision rests on the question $g(3) \leqq g(4)$ and can be settled analytically. The result is that $g(3)<g(4)$.

In the following, we assume case 4 , with

$$
10<1 / p \equiv e^{X},
$$


the choices being integers $m \geqslant 2$ for which $m(m+3) /(m-1)<1 / p$, and define $n(M \geqslant 5)$ as the greatest such integer:

$$
M(M+3) /(M-1,<1 / p \leqslant(M+1)(M+4) / M
$$

Regarding $g(m)$ as a function of a real variable $m$ on the interval $2 \leqslant m \leqslant M$, we find

$$
g^{-}(m)=c_{0} p^{2}\left(1-\frac{1}{p m^{2}}\right)\left\{1-\frac{1}{\sqrt{\frac{m-1}{m} \frac{1}{p}-(m-1)}}\right\} \text {. }
$$

Since $m^{2} /(m-1)$ is increasing with $m$ for $m>2$ we have $m^{2} /(m-1) \leqslant M^{2} /(M-1)<M(M+3) /(M-1)<1 / p$,

so that the bracket in (8) is positive, and $g^{-}(\mathrm{m})$ has the sign of $s(m) \equiv$ $1-\frac{1}{\mathrm{pm}^{2}}$ on $2 \leqslant \mathrm{~m} \leqslant \mathrm{M}$

$$
\text { But } s(2)=1-\frac{1}{4 p}<0 \text { since } 1 / p>4 \text {, and } s(M)=1-\frac{1}{\mathrm{pM}^{2}}>0 \text {, since by }(7) \text {, }
$$

$1 / p<(M+1)(M+4) / M<M^{2}$ for $M \geqslant 5$. Hence on the interval $[2, M], g(m)$ is decreasing up to

$$
\bar{m}=(1 / p)^{1 / 2}=e^{x / 2},
$$

is minimal at $\bar{m}$, and increases from $\bar{m}$ to $M$.

Thus the choice of optimal $\mathrm{m}$ lies between the immediately surrounding integers

$$
m_{1}<(1 / p)^{1 / 2}<m_{2} \leqslant M,
$$

depending on the inequality $g\left(m_{1}\right) \lesseqgtr g\left(m_{2}\right)$. If $(1 / p)^{1 / 2}=\bar{m}$ happens to be an integer, then $\bar{m}$ is of course the cheapest splitting number. In any case, the position of the splitting surface is given by (3), where $m$ is optimal. 